\title{
Aqueous Dispersions of Extraordinarily Small Polyethylene Nanoparticles
}

\author{
Ludmila Kolb, Vincent Monteil, Ralf Thomann and Stefan Mecking*
}

General methods and materials. Ethylene of 3.5 grade was supplied by Praxair. Demineralised water was distilled under argon. 2-propanol (p.a.) was degassed by four repeated freeze-thaw cycles. Chloranil (Aldrich) und sodium dodecyl sulfate (Fluka) were used without further purification. Potassium 4-(diphenylphosphino)benzenesulfonate (TPPMS) was synthesized as described in [1]. bis(1,5-cyclooctadiene) nickel was synthesized according to [2] (the compound is also available commercially, e.g. at Aldrich or Strem).

TEM investigations were carried out on a LEO 912 Omega apparatus using an acceleration voltage of $120 \mathrm{kV}$. Samples were stained with $\mathrm{RuO}_{4}$. For microtome cutting, the latex particles were embedded in nanoplast ${ }^{\circledR}$ (a hydrophilic melamine resin). Microtome cuts of ca. $50 \mathrm{~nm}$ thickness were prepared with a Reichert \& Jung Ultracut E microtome equipped with a $45^{\circ}$ diamond knife supplied by Diatome.

AFM experiments were performed with a Nanoscope III scanning probe microscope. The height and phase images were obtained simultaneously while operating the instrument in the tapping mode under ambient conditions. Images were taken at the fundamental resonance frequency of the Si cantilevers which was typically around $180 \mathrm{kHz}$. Typical scan speeds during recording were 0.3-1 line/s using scan heads with a maximum range of $16 \times 16 \mu \mathrm{m}$. The phase images represent the variations of relative phase shifts (i. e. the phase angle of the interacting cantilever relative to the phase angle of the freely oscillating cantilever at the resonance frequency) and are thus able to distinguish materials by their material properties (e.g. amorphous and crystalline polymers).

DSC was performed on a Perkin Elmer DSC 7 instrument or on a Pyris 1 DSC at a heating and cooling rate of $10 \mathrm{~K} \mathrm{~min}^{-1}$. $\mathrm{T}_{\mathrm{m}}$ data reported are local maxima of the second heats. DSC traces of polymer dispersions were obtained on 20 to 30 mg of dispersion with ca. $5 \%$ by weight polymer content.

NMR spectra were recorded on a Bruker ARX 300 instrument $\left({ }^{1} \mathrm{H}: 300 \mathrm{MHz} ;{ }^{13} \mathrm{C}: 75 \mathrm{MHz}\right) .{ }^{1} \mathrm{H}$ and ${ }^{13} \mathrm{C}$ NMR spectra were performed in 1,1,2,2-tetrachloroethane- $d_{2}$ at $122{ }^{\circ} \mathrm{C}$.

GPC analyses were carried out by Basell GmbH, Ludwigshafen on a Waters150 or GPC2000 instrument equipped with Shodex columns at $140^{\circ} \mathrm{C}$ in 1,2,4-trichlorobenzene. Data is referenced to linear polyethylene standards.

Dynamic light scattering on dispersions was performed on a Malvern particle sizer.

Catalyst Preparation. The preparation of catalyst was performed by standard Schlenk techniques under argon. Equal molar amounts of chloranil and TPPMS were dissolved in a given amount of 2-propanol (2 to $10 \mathrm{~mL})$. The obtained yellow solution was transferred to a 1.1-fold molar excess of bis(1,5-cyclooctadiene)nickel.

Polymerization Procedure. Polymerization was carried out in a mechanically stirred $250 \mathrm{~mL}$ pressure reactor equipped with a heating/cooling jacket, the temperature being controlled automatically by means of a sensor dipping into the reaction mixture. Ethylene consumption during polymerization was followed by a mass flow meter. The total volume of the liquid phase (water and 2-propanol) amounted to $100 \mathrm{~mL}$.

An aqueous solution of the surfactant was transferred to the reactor and heated to the specified temperature. The catalyst, prepared as described above, was then transferred to the polymerization reactor via a Teflon cannula. The reactor was flushed and pressurized with ethylene under vigorous stirring $(1000 \mathrm{rpm})$. After the specified reaction time, the reaction was stopped by releasing the gas pressure. The polymer latex formed was filtered through a funnel with glass wool, to determine and seperate any coagulate formed. For determination of the solids content, a specified portion of the latex was added to an excess of methanol. The precipitated polymers were isolated, washed with methanol, and dried in a vacuum.

$\left.{ }^{1}\right]$ O. Herd, A. Heßler, K. P. Langhans, O. Stelzer, W. S. Sheldrick, N. Weferling, J. Org. Chem. 1994, $475,99$.

$\left[{ }^{2}\right]$ R. A. Schunn, S. D. Ittel, M. A. Cushing, Inorg. Synth. 1990, $28,94$.

Konstanzer Online-Publikations-System (KOPS)

URL: http://www.ub.uni-konstanz.de/kops/volltexte/2007/4329/

URN: http://nbn-resolving.de/urn:nbn:de:bsz:352-opus-43296 


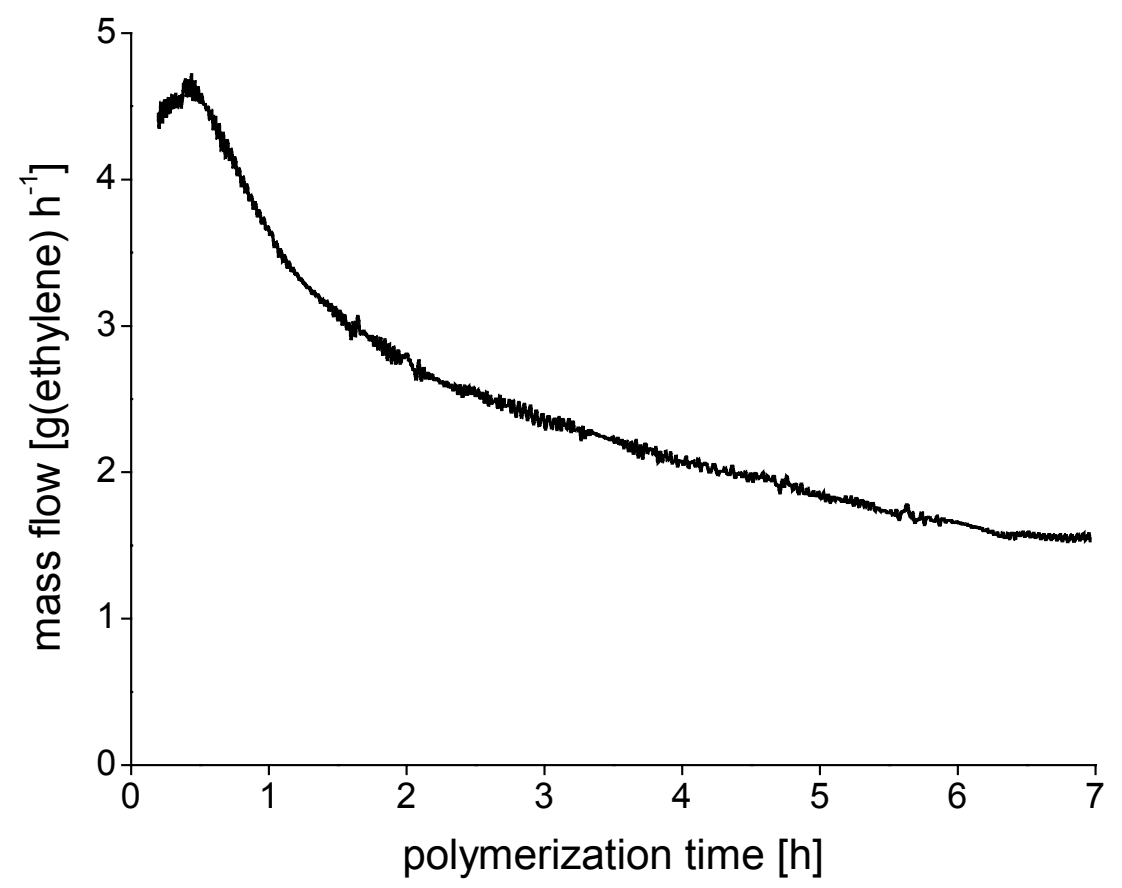

Figure S1. Ethylene consumption in a 7 hour experiment (polymerization conditions: 66 $\mu \mathrm{mol} \mathrm{Ni}, 40$ bar ethylene, $60^{\circ} \mathrm{C}$ ).

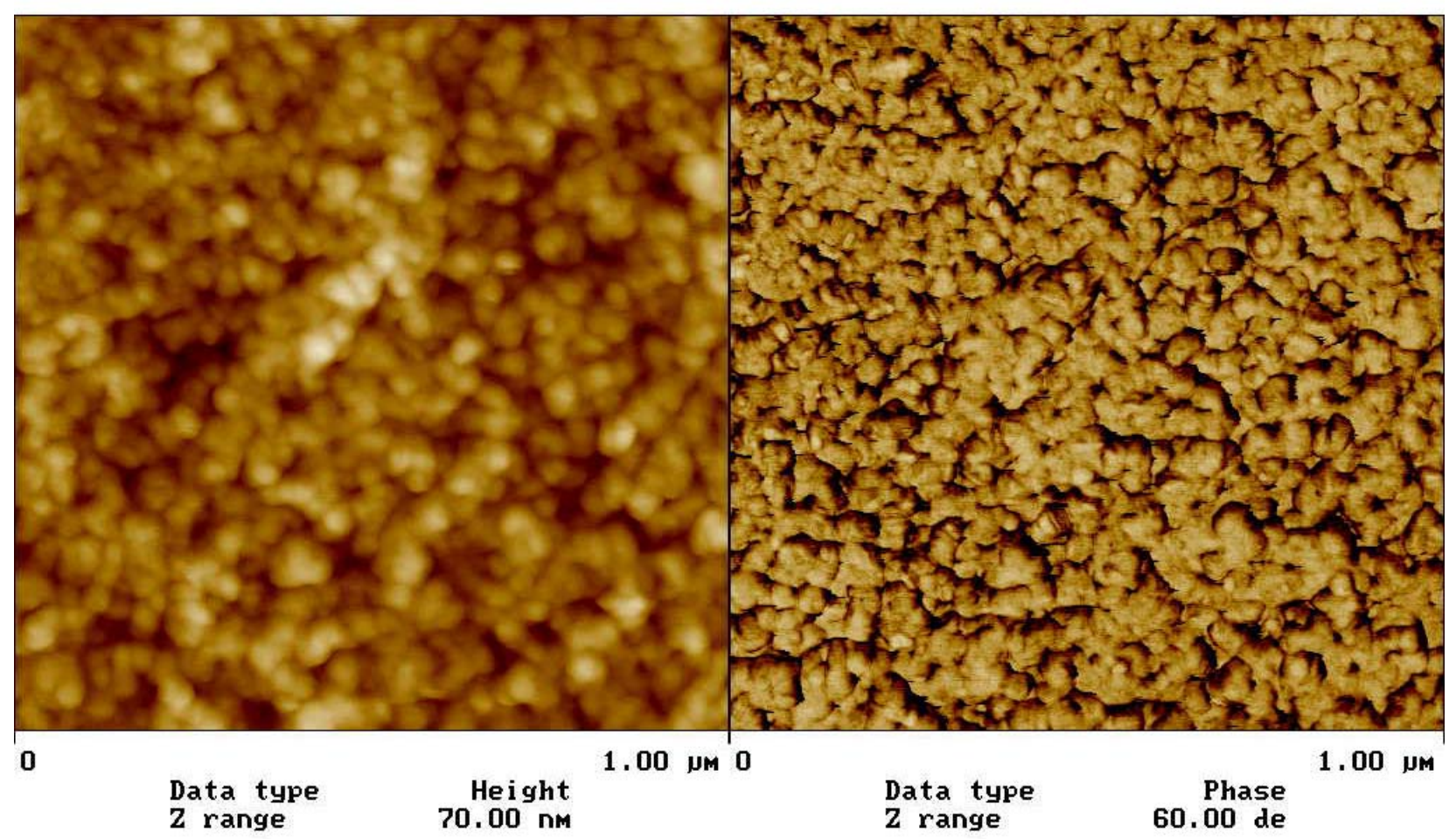

Figure S2. AFM images of polyethylene latices (left: height mode, right: phase mode). 


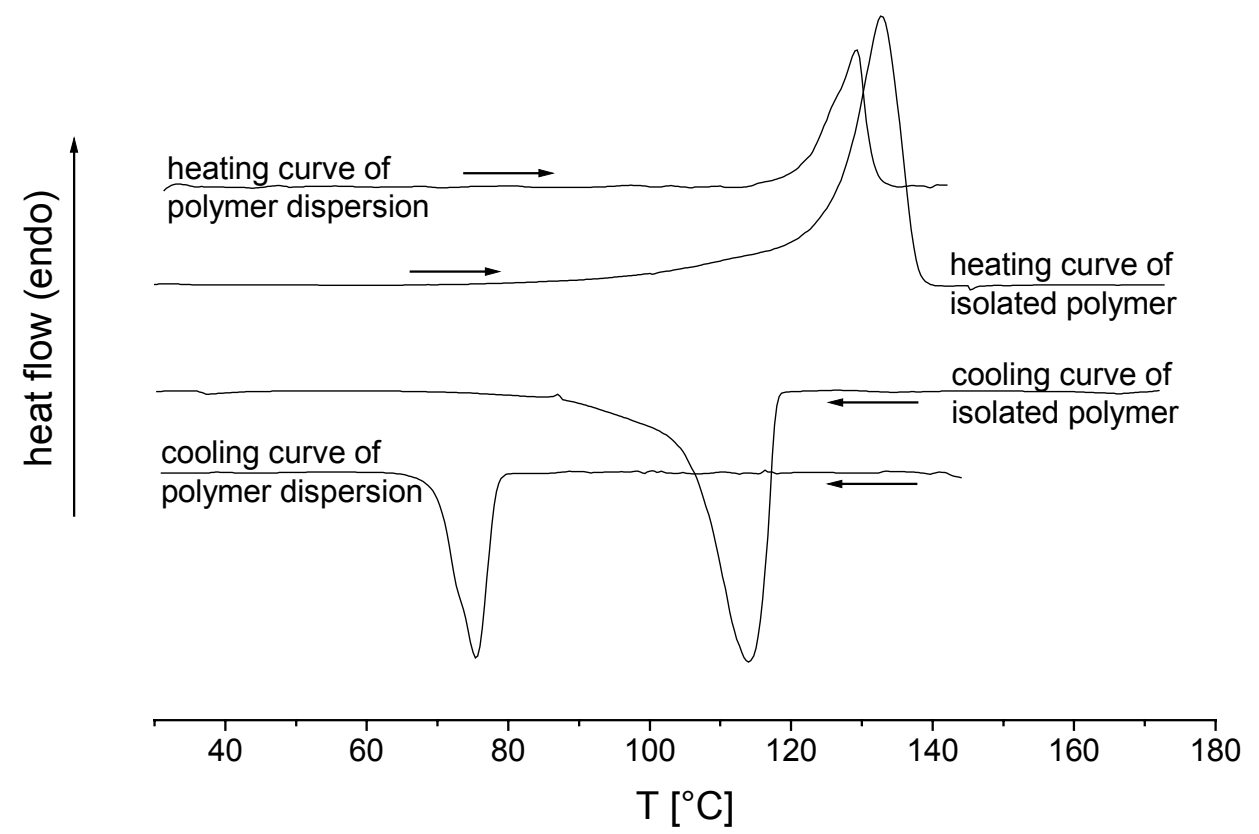

Figure S3. DSC traces of a polymer dispersion and of isolated bulk polymer

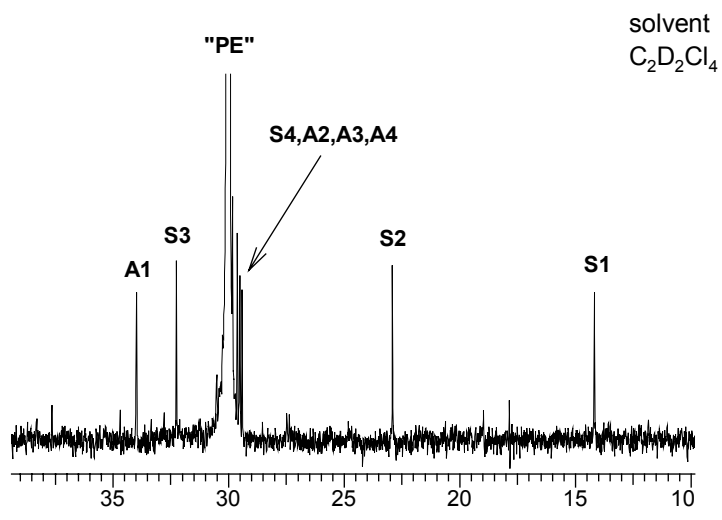

$$
\begin{array}{ccccc}
\text { s1 } & \text { s2 } & \text { s3 } & \text { S4 } & \text { "PE" } \\
\mathrm{CH}_{3}-\mathrm{CH}_{2}-\mathrm{CH}_{2}-\mathrm{CH}_{2}-\left(\mathrm{CH}_{2}\right)_{\mathrm{n}}- &
\end{array}
$$$$
\begin{array}{llllll}
\text { V1 } & \text { V2 } & \text { A1 } & \text { A2 } & \text { A3 } & \text { A4 }
\end{array}
$$$$
\mathrm{CH}_{2}=\mathrm{CH}-\mathrm{CH}_{2}-\mathrm{CH}_{2}-\mathrm{CH}_{2}-\mathrm{CH}_{2}-
$$

V2

V1

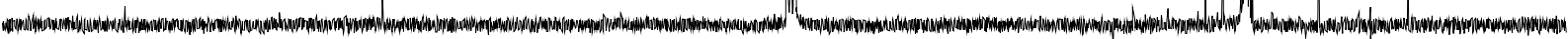

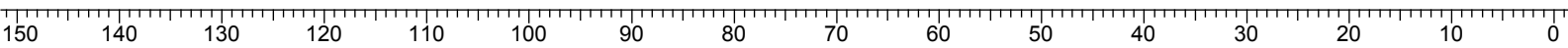

Figure S4. High-temperature ${ }^{13} \mathrm{C}$ NMR spectrum of polyethylene. 


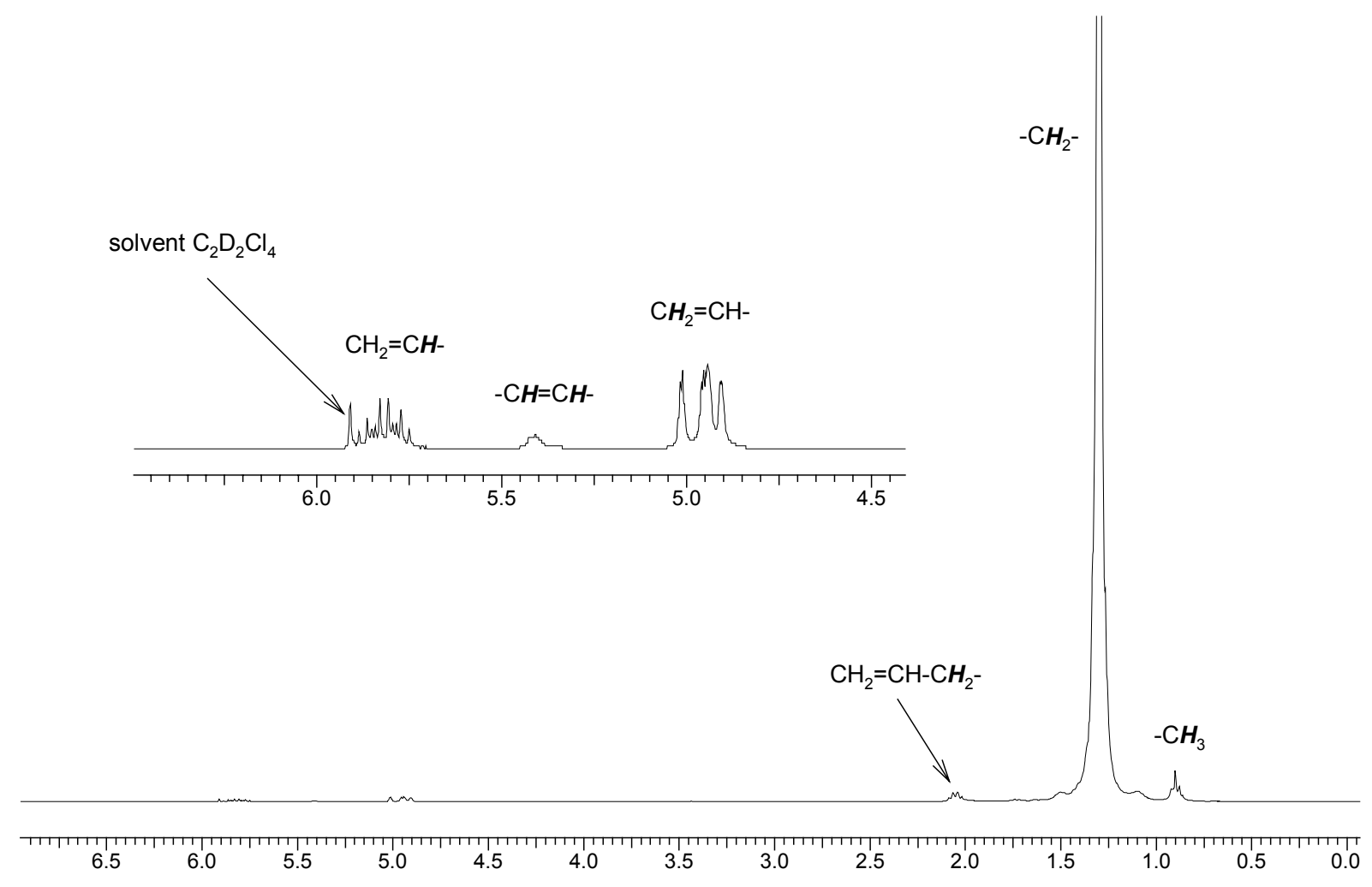

Figure S5. High-temperature ${ }^{1} \mathrm{H}$ NMR spectrum of polyethylene. 